\title{
INVESTIGAÇÃO DA INFLUÊNCIA DOS TRAÇOS DE PERSONALIDADE NO USO DE DIFERENTES ELEMENTOS MULTIMÍDIA
}

\section{INVESTIGATION OF THE INFLUENCE OF PERSONALITY TRAITS IN THE USE OF DIFFERENT MULTIMEDIA ELEMENTS}

\author{
Helena Macedo Reis - helena.macedo@ufpr.br \\ Universidade Federal do Paraná - Jandaia do Sul - Paraná - Brasil
}

Tiago Roberto Kautzmann - tkautzmann@gmail.com Universidade do Vale do Rio dos Sinos - São Leopoldo - Rio Grande do Sul - Brasil

Fernando Tiosso - fernando.tiosso@fatec.sp.gov.br Faculdade de Tecnologia de Taquaritinga -Taquaritinga -São Paulo-Brasil

Simone de Sousa Borges - simoneborges@utfpr.edu.br Universidade Tecnológica Federal do Paraná - Dois Vizinhos - Paraná - Brasil

Rodrigo Clemente Thom de Souza - thom@ufpr.br Universidade Federal do Paraná - Jandaia do Sul - Paraná - Brasil

Patrícia Augustin Jaques - pjaques@unisinos.br Universidade do Vale do Rio dos Sinos - São Leopoldo - Rio Grande do Sul - Brasil

DOI: 10.31510/infa.v17i2.892

Data de publicação: 18/12/2020

\section{RESUMO}

Em sistemas computacionais educacionais, os elementos de multimídia vêm sendo utilizados como ferramentas cognitivas importantes capazes de apoiar a compreensão de informações mais complexas. Porém, quando elaborados de maneira inadequada, os elementos de multimídia podem dificultar o entendimento de instruções, resultando em um alto esforço cognitivo pelos estudantes. Para minimizar este problema, estudos indicam princípios e/ou diretrizes que apoiam na elaboração de elementos multimídias mais efetivos durante o aprendizado, em que o processamento das informações é considerada. Entretanto, esses estudos não consideram aspectos individuais, como os traços de personalidade. Alguns estudos indicam que os traços de personalidade podem influenciar no processamento das informações, como por exemplo, a capacidade de processar informações visuais e auditivas ao mesmo tempo. Este artigo apresenta o planejamento, execução e os resultados de um experimento realizado para investigar como os traços de personalidade podem afetar no aprendizado, esforço cognitivo e sentimento de frustração pelos estudantes durante o uso de diferentes elementos multimídia. Observou-se que alguns estudantes demonstraram alto grau de ansiedade em utilizar o elemento multimídia exemplo-trabalhado. 
Palavras-chave: Traços de personalidade. Elementos de hipermídia. Carga cognitiva.

\begin{abstract}
In educational computer systems, media elements have been used as important cognitive tools, capable of supporting the understanding of more complex information. However, when elaborated inappropriately, the media elements can make it difficult to understand the instructions, resulting in a high cognitive effort by the students. To minimize this problem, studies adopt principles and / or guidelines that support the study of more effective multimedia elements during learning, in which information processing is used. However, these studies do not consider individual aspects, such as personality traits. Some studies indicate that personality traits can influence information processing, such as the ability to process visual and auditory information at the same time. This article presents the planning, execution and results of an experiment carried out to investigate how personality traits can affect students' learning, cognitive effort and feelings of frustration when using different multimedia elements. It was observed that some students showed a high degree of anxiety and impatience in using the example worked.
\end{abstract}

Keywords: Personality traits. Multimedia elements. Cognitive load.

\title{
1 INTRODUÇÃO
}

Em ambientes de aprendizagem, a multimídia vem sendo usada como uma ferramenta cognitiva capaz de apoiar e engajar os estudantes e professores em tarefas complexas. Esses elementos, tais como texto, vídeos, imagens, entre outros, podem influenciar no aprendizado (SHIMOMURA; HVANNBERG; HAFSTEINSSON, 2013), fazendo com que o entendimento das informações mais complexas seja facilitado ou dificultado. Quando esses elementos não são devidamente projetados, os estudantes podem sentir dificuldades, direcionando a sua atenção para o entendimento da estruturação material ao invés do seu conteúdo. Um grande número de informações pode ocultar os processos de aprendizado, requerendo que os estudantes realizem um alto esforço cognitivo (MAYER; MORENO, 2003; REIS ET AL., 2012), podendo aumentar os sentimentos de frustração e tédio.

É comum os estudantes tomarem decisões e escolherem estratégias erradas para a resolução de problemas, quando possuem alto esforço cognitivo. Esse esforço é resultado de 
quando a carga cognitiva ${ }^{1}$ é utilizada até a sua exaustão, chamada de sobrecarga cognitiva (ALVES ET AL., 2017). A sobrecarga afeta os processos cognitivos, como memória, atenção e a percepção, resultando em erros cognitivos e sócio cognitivos (KNIPPENBERG; DIJKSTERHUIS; VERMEULEN, 1999).

Dentro do ambiente educacional, a sobrecarga cognitiva pode impactar negativamente no desempenho escolar, podendo interferir em uma avaliação e tomada de decisão de quais estratégias de aprendizagem adotar. Por exemplo, a sobrecarga contribui para a dificuldade de processamento das instruções e o entendimento do assunto, aumentando a probabilidade de cometer muitos erros e consequentemente sentir frustração ou tédio.

Os resultados do estudo de Matthews (2008) sugerem que os traços de personalidade podem influenciar em como as informações são processadas pelos indivíduos e consequentemente o esforço cognitivo. Nesse estudo, o autor apontou que indivíduos com traços de personalidade de extroversão gerenciam de maneira mais apropriada a memória a curto e longo prazo, além de trabalharem de maneira mais adequada a atenção em diferentes canais (e.g. canal auditivo e canal visual) comparado com indivíduos com traços de personalidade de neuroticismo. Dessa forma, temos o seguinte questionamento: Os elementos de multimídia influenciam no aprendizado de estudantes com traços de personalidade de extroversão e neuroticismo?

Para investigar quais os tipos de elementos de multimídia são mais adequados para o uso da cognição considerando as características individuais do estudante, como o traço de personalidade e o conhecimento prévio, foi desenvolvido um experimento com estudantes do ensino fundamental de um colégio particular brasileiro. $\mathrm{O}$ artigo encontra-se dividido da seguinte forma: na seção 2 são comentados os trabalhos relacionados sobre a elicitação de requisitos por análise de documentos. A seção 3 apresenta o método aplicado para buscar responder a questão de pesquisa. A seção 4 descreve a análise e discussão. E finalmente, nas seções 5 e 6 temos as ameaças à validade e a conclusões da pesquisa realizada, respectivamente.

\footnotetext{
${ }^{1}$ A carga cognitiva refere-se à quantidade de instruções processadas pela memória de trabalhado dos estudantes durante o processo de aprendizagem
} 


\section{TRABALHOS RELACIONADOS}

A Teoria da Aprendizagem Multimídia (TAM) proposta por Mayer e Moreno (2003) indica que o uso de elementos verbais e visuais juntos melhoram o uso da memória de trabalho. Os resultados do estudo indicaram que os estudantes possuíam mais dificuldades em memorizar o conteúdo aprendido quando eram ensinados por métodos exclusivamente verbais, como fala e escrita. Desta forma, a TAM sugere que o uso de imagens e textos de forma conjunta durante o ensino é mais efetivo e os estudantes conseguem aprofundar mais seus conhecimentos, comparado com o uso de materiais que apresentam somente texto.

Seguindo a ideia proposta por Mayer e Moreno (2003), o estudo de Lyra e Isotani (2017) investigou se o uso de exemplos apresentados em figuras (infográficos) como materiais de aprendizagem era mais efetivo e adequado comparado aos materiais tradicionais, como gráficos e textos. Após um experimento empírico com 74 alunos de graduação sobre conteúdo do meio-ambiente, os resultados apontaram que o uso dos infográficos (imagens e texto) apresentaram benefícios comparado com os materiais tradicionais em termos de aprendizado. Ao utilizar infográficos, os estudantes demoraram menos tempo para aprender o conteúdo.

O exemplo trabalhado é outro tipo de elemento de multimídia que pode ser apresentado aos estudantes além de imagens e textos. Ele é apresentado em formato passo a passo, acompanhando o estudante em uma linha de raciocínio de acordo com a TCC (Teoria da Carga Cognitiva). Neste caso, a intenção é fazer com que o estudante use menos a sua carga cognitiva, evitando a sobrecarga cognitiva com várias informações (SALDEN ET AL., 2010; SCHWONKE ET AL., 2009). Os estudos de Salden et al. (2010) e Schwonke et al. (2009) sugerem que o uso de exemplos trabalhados é mais eficiente em estudantes iniciantes no assunto comparado aos estudantes que possuem mais conhecimento.

\section{MÉTODO}

\subsection{Objetivos e Hipóteses}

O propósito deste experimento é avaliar quais tipos de elementos de multimídia fornecem melhor entendimento e causam menos esforço cognitivo para cada traço de 
personalidade, especialmente para o traço de extroversão e neuroticismo. Os tipos de elementos de multimídia que serão investigados são texto, exemplo trabalhado e figura. A partir dessa abordagem, foi formulada a questão de pesquisa principal:

QP 1: Os elementos de multimídia influenciam no aprendizado de estudantes com traços de personalidade de extroversão e neuroticismo?

Para a questão de pesquisa 1, temos formalmente as hipóteses:

- Hipótese Nula: Não existe diferença entre o aprendizado por figuras, exemplo trabalhado e texto, para cada traço de personalidade.

$\mathrm{H} 0=$ Aprendizado $_{(\text {figura })}=$ Aprendizado $_{(\text {exemplo trabalhado })}=$ Aprendizado $_{(\text {texto })}$

- Hipótese Alternativa: Existe diferença entre o aprendizado por figura, exemplo trabalhado e texto, para cada traço de personalidade.

$\mathrm{H} 1=$ Aprendizado $_{\text {(figura) }} \neq$ Aprendizado $_{\text {(exemplo trabalhado) }} \neq$ Aprendizado(texto) $_{\text {(t) }}$

Além da investigação do ganho de aprendizagem, também foi investigada à influência da variável de esforço cognitivo ao uso de diferentes tipos de elemento de multimídia. Assim, o objetivo é investigar qual elemento de multimídia o estudante sentiu menos esforço cognitivo (QP2: Os diferentes tipos de elementos de multimídia influenciam no esforço cognitivo?). Formalmente, tem-se:

- Hipótese Nula: Não existe diferença entre o esforço cognitivo dos estudantes na utilização de figura, exemplo trabalhado e texto.

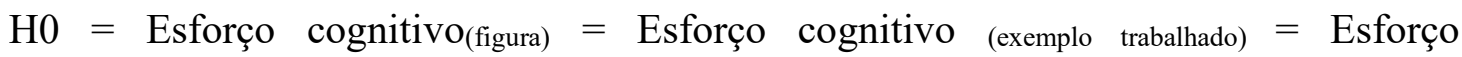
cognitivo(texto)

- Hipótese Alternativa: Existe diferença entre o esforço cognitivo dos estudantes na utilização de figura, exemplo trabalhado e texto.

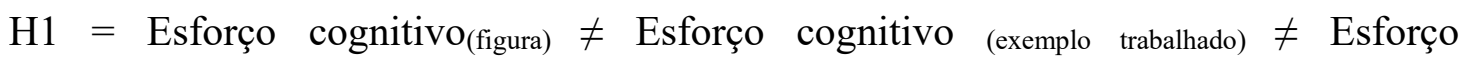
$\operatorname{cognitivo}_{(\text {texto) }}$

E por fim, foi investigado se os diferentes elementos de multimídiam influenciam no sentimento de frustração pelos estudantes (QP3: Os diferentes tipos de elementos de multimídia influenciam no sentimento de frustração?). Desta maneira, temos a terceira hipótese:

- Hipótese Nula: Não existe diferença entre a frustração dos estudantes na utilização de figura, exemplo trabalhado e texto.

$\mathrm{H} 0=$ Frustração $_{\text {(figura) }}=$ Frustração (exemplo trabalhado) $^{=}$Frustração (texto) 
- Hipótese Alternativa: Existe diferença entre a frustração dos estudantes na utilização de figura, exemplo trabalhado e texto.

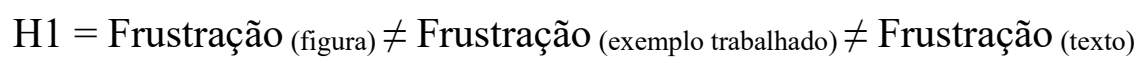

\subsection{Participantes}

Participaram do experimento 65 estudantes de um colégio particular na cidade de Araraquara, no estado de São Paulo. Os estudantes foram três turmas do $7^{\circ}$ ano (Média $\approx 13.5$ anos) com noções básicas sobre equações do primeiro grau. Ao final do experimento, os dados de 53 sujeitos puderam ser considerados por cumprir todas as fases: Pré-teste (Fase A), Intervenção (Fase B) e Pós-teste (Fase C).

\subsection{Materiais}

Os materiais utilizados para ao experimento foram: (i) teste de álgebra para o conhecimento prévio (pré-teste) em papel, (ii) questionário para identificar o traço de personalidade do estudante, (iii) questões de álgebra no STI PAT2Math com diferentes tipos de elementos de multimídia, (iv) teste em papel para verificar o conhecimento pós intervenção (pós-teste) e (v) teste de esforço cognitivo.

O teste de conhecimento de álgebra (pré e pós-teste) tem como objetivo identificar o nível de conhecimento que o estudante possui em álgebra, sem o uso do computador. Desta maneira, os resultados não foram impactados por alguma eventual falta de habilidade ou conhecimento computacional. Além disso, se o estudante possuir alto nível de conhecimento em álgebra por esse teste e baixo nível no conhecimento em álgebra usando os elementos de multimídia, poderia indicar que os elementos de multimídia não foram elaborados de forma adequada. Os estudantes responderam por meio da caneta e papel 20 questões de álgebra, divididas em níveis fáceis (3), intermediários (4), avançados (6) e experts (7).

O questionário usado para identificar o traço de personalidade foi baseado no estudo de Barbosa (2009). Nele, o autor propõe 20 questões que podem ser respondidas na escala de 1 (Discordo totalmente) a 5 (Concordo totalmente). Cada pergunta pode ser categorizada em um dos cinco grupos: amabilidade, extroversão, realização, abertura à mudanças e neuroticismo. No final, as respostas foram somadas, e foi verificado qual grupo foi mais pontuado. 


\section{[NTERFFAET TECNOLOGGCA}

Os resultados do teste de álgebra disponível em diferentes tipos de elementos de multimídia foram comparados com o teste de conhecimento de álgebra. Esse teste foi disponibilizado eletronicamente pelo sistema PAT2Math, em que as respostas são verificadas por um tutor virtual. A cada resposta (passo da resolução), o tutor virtual fornece feedback mínimo (certo ou errado) ao estudante de forma imediata. De acordo com os resultados de estudos anteriores, acredita-se que o uso de elementos visuais em conjunto com elementos textuais pode ser benéfico para o aprendizado. Segundo Mayer e Moreno (2003), essa premissa pode ser aplicada tanto para materiais impressos quanto para materiais digitais, além de ser independente do conteúdo lecionado. Entretanto, não foram encontrados estudos que investigam se esses princípios são eficazes para diferentes tipos de personalidade e conhecimento prévio de álgebra. Devido a isso, para esse experimento, foram escolhidos os seguintes tipos de elementos de multimídia:

- Texto: o conteúdo é explicado somente com palavras;

- Figura: o conteúdo é explicado por meio de texto e imagens, sem qualquer animação. Algumas figuras explicativas foram adicionadas e;

- Exemplo trabalhado: o exercício é explicado e apresentado passo a passo com o auxílio do tutor virtual.

Cada material apresentou a resolução de um exercício como exemplo. Para determinar o nível de complexidade do conteúdo, foi adotado que para cada exercício seria mostrado a sua resolução passo a passo, além de relembrar os conceitos estudados em sala de aula com os professores. Esses conceitos foram baseados na sistematização proposta por Ponte, Branco, Matos (2009), em que investiga os erros e as dificuldades dos estudantes na simplificação de expressões algébricas e na resolução de equações do $1^{\circ}$ grau.

A seleção dos exercícios foi baseada nos exercícios propostos no sistema PAT2Math, sendo divididos em níveis básico (4 exercícios), intermediário (4 exercícios), avançado (4 exercícios) e expert (3 exercícios). Após a seleção dos exercícios e quais conceitos deveriam ser discutidos de acordo com os erros e dificuldades mais encontradas, cada elemento de multimídia acima foi elaborado de acordo com os doze princípios de Mayer e Moreno (2003).

Ao término de cada nível (i.e. básico, intermediário, avançado e expert), era apresentado o formulário para identificar o esforço cognitivo do estudante. Nele, o estudante precisava indicar a frequência de cada subescala do NASA TLX (HART; STAVELAND, 1988) e o seu peso. 


\subsection{Procedimento}

Os sujeitos participantes do experimento foram separados aleatoriamente em três grupos de aproximadamente 65 alunos entre as três turmas para utilizarem texto, exemplo trabalhado e figuras. $\mathrm{O}$ experimento consistiu em três fases (Figura 1): Fase A (Pré-teste), Fase B (Intervenção) e Face C (Pós-teste).

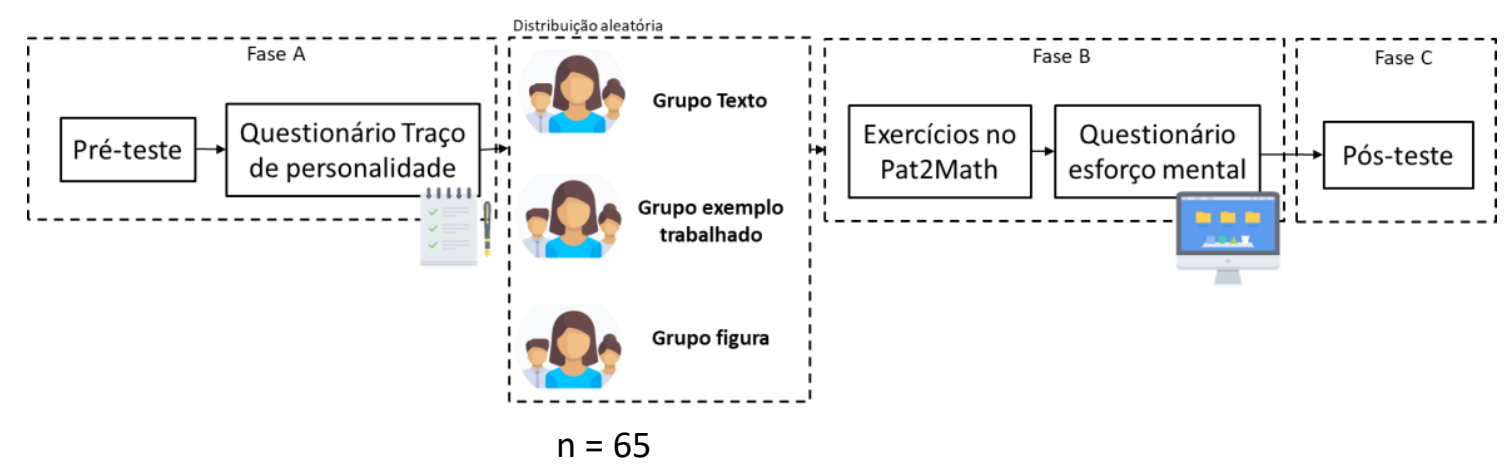

Figura 1 - Procedimento do experimento

A execução do experimento ocorreu em três dias, distribuídos em 1 dia para cada semana. A Fase A (pré-teste) foi realizada em sala de aula, utilizando o papel e lápis. Primeiramente, os sujeitos receberam orientações oralmente sobre os objetivos da atividade e sigilos dos dados. Em seguida, foi solicitado que os sujeitos respondessem o questionário do traço de personalidade. Posteriormente, os sujeitos realizaram o teste de conhecimento prévio, que consistiu em 20 questões de álgebra, divididas em níveis fáceis (3), intermediários (4), avançados (6) e experts (7). Cada uma dessas questões deveria ser respondida de forma discursiva. Esse teste tinha o objetivo de mensurar o conhecimento em álgebra dos sujeitos, para ser posteriormente comparado com os resultados obtidos com o teste de álgebra usando diferentes elementos de multimídia.

Depois de uma semana, os estudantes realizaram as atividades da Fase B (intervenção). Nela, os sujeitos executaram todas as atividades individualmente em um laboratório de informática, com apoio do lápis e papel para rascunho. Os sujeitos foram distribuídos aleatoriamente para resolver os exercícios em álgebra. As três turmas resultaram em 22 sujeitos para cada tipo de elemento de multimídia (exceto o grupo de exemplotrabalhado). Antes de cada exercício, foi apresentada uma explicação de 15 exercícios em três diferentes elementos de multimídia: texto, exemplo trabalhado e figura. Os sujeitos foram 
responsáveis por responder 15 exercícios de níveis fáceis, intermediário, avançado, e expert usando um tipo de elemento de multimídia. Ao término de cada nível, foi solicitado que o sujeito autodeclarasse o seu esforço cognitivo com a atividade proposta com o determinado elemento de multimídia.

Por fim, após uma semana, a Fase C (pós-teste) os sujeitos realizaram o pós-teste que consistiu em 20 questões de álgebra semelhante ao teste de conhecimento prévio do pré-teste, dividas em níveis fáceis (3), intermediários (4), avançados (6) e experts (7). Cada uma dessas questões deveria ser respondida de forma discursiva.

Ao fim da execução, os dados coletados foram tabulados e analisados. Dentre os 65 alunos participantes, os dados de 53 sujeitos (28 do sexo feminino e 25 do sexo masculino) puderam ser considerados; os outros não cumpriram as três fases do experimento.

\section{ANÁLISE E DISCUSSÃO DOS RESULTADOS}

Para investigar as hipóteses foi realizado um teste de normalidade utilizando o teste Shapiro-Wilk para determinar se o conjunto de dados de uma dada variável é considerada uma distribuição normal ou não. Posteriormente, para analisar a primeira questão de pesquisa (QP1: Os elementos de multimídia influenciam no aprendizado de estudantes com traços de personalidade de extroversão e neuroticismo?) foi aplicado o teste-t comparando a diferença entre as notas do pré-teste e do pós-teste. O teste-t não mostrou diferença estatística significativa para nenhum dos elementos de multimídia para o traço de personalidade neuroticismo, sendo Figura com $p$-value $=0.707$, Exemplo-trabalhado com $p$-value $=0.561 \mathrm{e}$ Texto com $p$-value $=0.304$. Também não foi encontrada diferença significativa com o traço de personalidade extroversão, sendo $p$-value $=0.470$ (Figura), p-value $=0.553$ (Exemplotrabalhado) e $p$-value $=0.783$ (Texto). Desta forma, os resultados sugeriram que a hipótese nula (Hipótese Nula: Não existe diferença entre o aprendizado por figuras, exemplo trabalhado e texto, para cada traço de personalidade) não deve ser rejeitada.

Para a segunda questão de pesquisa (QP2: Os diferentes tipos de elementos de multimídia influenciam no esforço cognitivo?). Os diferentes tipos de elementos de multimídia influenciam no esforço cognitivo foram utilizados os testes One-way Anova e o teste Tukey . Uma análise de variância simples (One-way Anova) tem como objetivo rejeitar ou não a hipótese de igualdade de médias populacionais de diversos grupos, porém não 


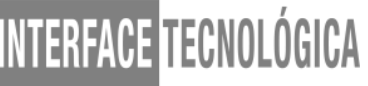

determina quais grupos têm médias estatisticamente diferentes. O teste de Tukey compara médias duas a duas.

No teste One-way Anova mostra que não houve significância estatística ( $p$-value $=$ 0,214). No teste de Tukey tivemos os resultados: Figura-Exemplo Trabalhado ( $p$-value= 0,2038), TextoExemplo Trabalhado ( $p$-value $=0,4063)$ e Texto-Figura $(p$-value $=0,8831)$.

Os resultados podem ser visualizados na Figura 2, que demonstra que esforço cognitivo de quem teve contato com figuras foi menor do que aqueles que tiveram contato com exemplo trabalhado e texto. Entretanto, não houve diferença estatística do esforço cognitivo e o aprendizado por personalidade. Assim, a hipótese nula (Hipótese Nula: Não existe diferença entre a esforço cognitivo dos estudantes na utilização de figura, exemplo trabalhado e texto.) não deve ser rejeitada.

Figura 2 - Relação entre elementos de multimídia e a frustração

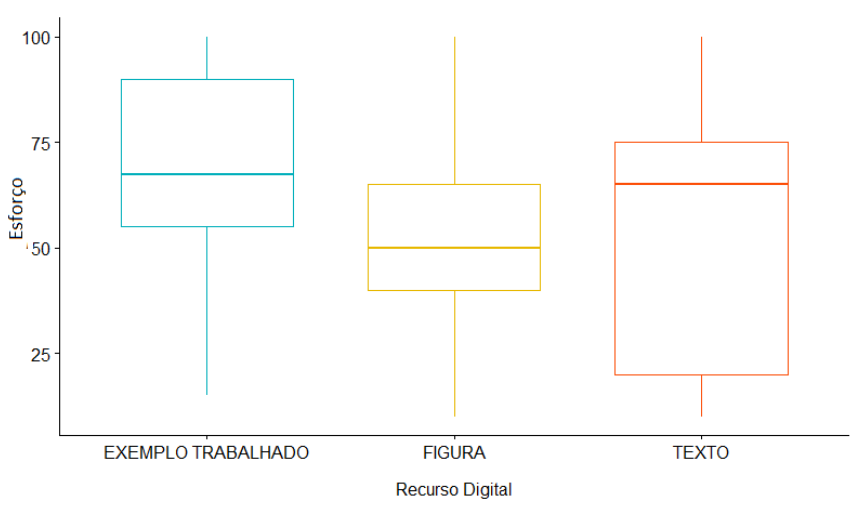

Fonte: Próprio autor

Os elementos de multimídia também foram analisados em relação a frustração (Figura 3). O teste One-way Anova demonstra que não houve significância estatística ( $p$ value $=0,179$ ). Além disso, o teste de Tukey demonstra que não há diferenças entre os três elementos de multimídia, Figura-Exemplo trabalhado ( $p$-value $=0,220)$, Texto-Exemplo trabalhado $(p$-value $=0,981)$ e Figura-Texto $(p$-value $=0,267)$. 
Figura 3 - Relação entre elementos de multimídia e a frustração

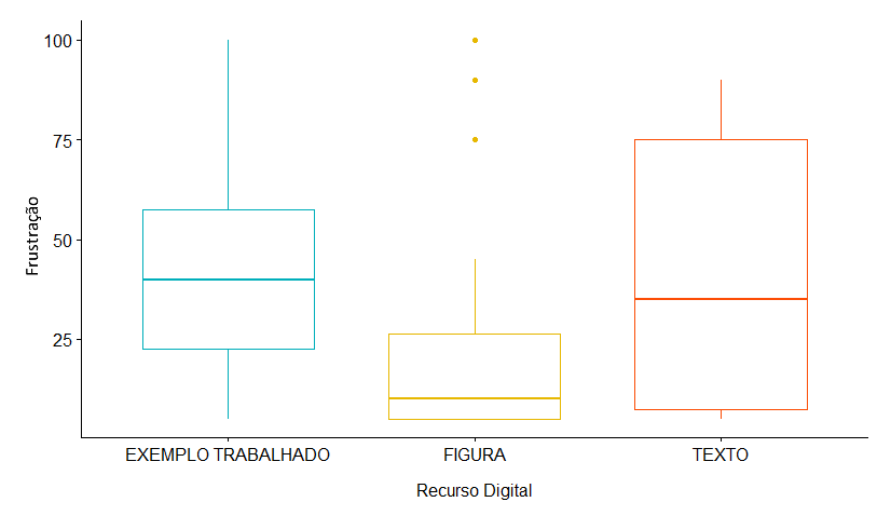

Fonte: Próprio autor

\section{AMEAÇAS À VALIDADE}

Uma possível ameaça à validade dos resultados é a representatividade da amostra, uma vez que indivíduos que participaram do estudo são estudantes do $7^{\circ}$ ano do ensino fundamental de uma única escola. Desta forma, não é possível generalizar os resultados para toda a população estudantil. Do ponto de vista estatístico, este problema pode ser contornado com a repetição com maiores número de participantes. Outra ameaça a ser considerada é a utilização do PAT2Math, pois os estudantes não possuíram tempo hábil para se adaptarem ao sistema, podendo impedir que alguns alunos completassem os exercícios. Embora a escolha dos exercícios se baseou nas versões estáveis do PAT2Math, elas podem não sido adequadas ao perfil dos participantes.

\section{CONCLUSÃO}

Os elementos de multimídia vêm sendo usados como ferramentas cognitivas para auxiliar no processo de ensino-aprendizagem mediados pelo computador. Porém, quando não 


\section{[NTERFFAET TECNOLOGGCA}

elaborados de maneira adequada, podem prejudicar a compreensão de informações pelos estudantes e levarem a sentir frustração e tédio (REIS; JAQUES; ISOTANI, 2017).

Apesar de Mayer e Moreno (2003) apresentarem 12 princípios para a elaboração de elementos multimídia para um aprendizado efetivo por meio da Teoria da Aprendizagem Multimídia (TAM), a teoria não considera características individuais de cada estudante. Por outro lado, o estudo de Matthews (2008) indica que os diferentes traços de personalidade podem influenciar no processamento das informações. Os resultados apontaram que indivíduos com traços de personalidade de neuroticismo possuíram dificuldades em realizar algumas funções cognitivas, como por exemplo, dividir a atenção entre elementos verbais e matemáticos devido a ansiedade prejudicar a performance das tarefas. Além disso, o estudo apontou que indivíduos com traço de personalidade de neuroticismo teve maior esforço cognitivo e um gerenciamento comprometido da memória de trabalho devido a ansiedade e impaciência.

Esses resultados podem corroborar com a observação durante a execução do experimento. Apesar da literatura acreditar que o uso dos exemplos trabalhados seja benéfico para o uso da carga cognitiva, os estudantes que utilizaram esse tipo de elemento de multimídia não terminavam de ler o exemplo. Os estudantes apresentaram ser agitados e ansiosos, não concluindo toda a leitura requisitada e clicavam rapidamente para o próximo passo do exemplo trabalhado.

Apesar dos testes estatísticos mostrarem-se inconclusivos, não sendo possível rejeitar nenhuma das hipóteses nulas formuladas devido aos altos valores de $p$ obtidos (todos próximos a 1), é possível inferir que o número pequeno de sujeitos presentes na amostra e o conhecimento prévio do estudante, pode ter influenciado nestes resultados. Pelos resultados obtidos no experimento, constatou-se que os tipos de elementos de multimídia não estão relacionados com o traço de personalidade do estudante ou o esforço cognitivo. O conhecimento em álgebra da maioria dos sujeitos estava abaixo da média das amostras, tendo vários sujeitos recebendo a nota mínima no pré-teste. Além disso, por serem crianças do $7^{\circ}$ ano, as equações de primeiro grau estão sendo introduzidas no conteúdo escolar, não tendo tempo suficiente para poderem aprender mais profundamente sobre conceitos do assunto tratado.

Desta maneira, acreditamos que a execução do experimento em crianças com conhecimento um pouco mais elevado em álgebra, redução de questões para evitar o desgaste 
e o aumento no número de participantes, possa fornecer dados mais significativos ao experimento.

\section{REFERÊNCIAS}

ALVES, M. V. C et al. As dimensões da carga cognitiva e o esforço mental. Revista Brasileira de Psicologia, v. 1, n. 4, 2017.

BARBOSA, A.Modelo hierárquico de fobias infanto-juvenis: testagem e relação com osestilos maternos. Tese (Doutorado) - Tese de doutorado apresentada ao Programa de PósGraduação Programa Integrado de Doutorado em Psicologia Social (UFPB/UFRN)), 2009.

HART, S. G.; STAVELAND, L. E. Development of nasa-tlx (task load index): Results of empirical and theoretical research. In: Advances in psychology. [S.1.]: Elsevier, 1988. v. 52, p. 139-183.

KNIPPENBERG, A. V.; DIJKSTERHUIS, A.; VERMEULEN, D. Judgement and memory of a criminal act: The effects of stereotypes and cognitive load. European Journal of Social Psychology, Wiley Online Library, v. 29, n. 2-3, p. 191-201, 1999.

LYRA, K.; ISOTANI, S. Impacto do uso de infográficos como materiais de aprendizagem e suas correlações com satisfação, estilos de aprendizagem e complexidade visual. In: Anais dos Workshops do Congresso Brasileiro de Informática na Educação. [S.1.: s.n.], 2017. v. 6, n. 1, p. 46.

MAYER, R.; MORENO, R. Nine Ways to Reduce Cognitive Load in Multimedia Learning. In: Educational Psychologist. [S.1.: s.n.], 2003. v. 38, p. 43-52. ISBN 0046-1520.

MATTHEWS, Gerald. Personality and information processing: A cognitive-adaptive theory. Handbook of personality theory and assessment, v. 1, p. 56-79, 2008.

PONTE, J. P. d.; BRANCO, N.; MATOS, A. Álgebra no ensino básico. In: . [S.1.]: MEDGIDC, 2009.

REIS, H. M. et al. Towards reducing cognitive load and enhancing usability through a reduced graphical user interface for a dynamic geometry system: An experimental study. In: IEEE. Multimedia (ISM), 2012 IEEE International Symposium on. [S.1.], 2012. p. 445-450.

REIS, H. M.; JAQUES, P. A.; ISOTANI, S. Sistemas tutores inteligentes que reconhecem o estado emocional do estudante: Um mapeamento sistemático. intelligent tutors systems that recognize the student's emotional state: A systematic mapping. Research and Innovation in Brazilian Education, 2(2), pp.101-114. Lemann Center, Stanford: Palo Alto, 2017.

SALDEN, R. J. et al. The expertise reversal effect and worked examples in tutored problem solving. Instructional Science, Springer, v. 38, n. 3, p. 289-307, 2010. 
SCHWONKE, R. et al. The worked-example effect: Not an artefact of lousy control conditions. Computers in Human Behavior, Elsevier, v. 25, n. 2, p. 258-266, 2009.

SHIMOMURA, Y.; HVANNBERG, E. T.; HAFSTEINSSON, H. Haptic cues as a utility to perceive and recognize geometry. Universal Access in the Information Society, Springer, v. 12, n. 2, p. 125-142, 2013. 